
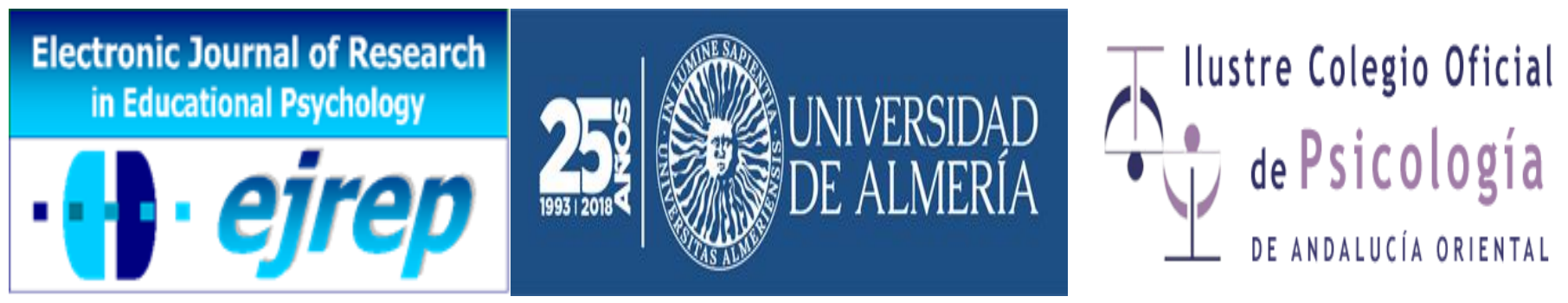

\title{
Agresión y victimización: la percepción del alumnado y factores asociados
}

\section{Cristina Ma García-Fernández, Eva Ma Romera-Félix, Francisco Córdoba-Alcaide, Rosario Ortega-Ruiz}

Departamento de Educación, Universidad de Córdoba, Córdoba

\section{España}

Correspondencia: Cristina $M^{a}$ García Fernández. Facultad de Ciencias de la Educación, Avd/ San Alberto Magno s/n, C.P:14071. España. E-mail: cristina.garcia@uco.es

(C) Universidad de Almería and Ilustre Colegio Oficial de la Psicología de Andalucía Oriental (Spain) 


\section{Resumen}

Introducción. La investigación sobre el bullying, así como de los factores protectores o de riesgo que se presenten en la escuela continúa siendo un área de estudio relevante en investigación educativa y también en la práctica escolar. Determinadas características personales y contextuales se han considerado claves para la prevención de la violencia escolar.

Método. El presente trabajo trata de examinar la relación existente entre la victimización y la agresión y el impacto que en ambos procesos psicológicos tienen ciertas variables del contexto directo de los escolares. Para ello se encuestó a 1278 escolares (47.7\% chicas) del sur de España (Andalucía), de edades comprendidas entre los 10 a 14 años $(M=11.11 ; D T=0.754)$.

Resultados. Análisis de regresión lineal mostraron el poder explicativo de factores protectores como el ajuste social o el ajuste a las normas ante ambos, la percepción de victimización y agresión injustificada. Factores individuales, como el género y personales como la autoestima marcan diferencias en la percepción de implicación en ambos roles, junto a otros.

Discusión y conclusiones. El foco de este artículo se pone en el papel de los factores de contexto inmediato.

Palabras Clave: agresión, victimización, factores personales, factores contextuales. 


\begin{abstract}
Introduction. Research on bullying, as well as on protective or risk factors in both phenomena continues to be a relevant area of study at the research level and with important repercussions for educational practice. Personal and contextual characteristics have been considered keys for the prevention of school violence.
\end{abstract}

Method. The aim of this study is to examine the relation between victimization and aggression roles and the impact that in both psychological processes have certain variables of the direct context of the students. For this purpose, 1278 students we surveyed $(47.7 \%$ girls $)$, from southern Spain (Andalusia), aged 10 to 14 years $(M=11.11 ; S D=0.754)$.

Results. Linear regression analysis indicated the explanatory power of protective factors such as social adjustment or norms adjustment, on the perception of victimization and unjustified aggression. Individual factors such as gender and personal as self-esteem mark differences in the perception of involvement in both roles, along with others.

Discussion and Conclusion. The focus of this article is put on the role of immediate context factors.

Keywords: Aggression, victimization, personal factors, contextual factors, Elementary School. 


\section{Introducción}

La vida social y las relaciones entre niños y niñas en las escuelas de educación básica (Primaria y primeros tramos de Secundaria en los países en que ésta está generalizada) se ha convertido en uno de los grandes retos educativos ante la necesidad de estimular la construcción de competencias sociales y emocionales, más allá de las puramente académica. Este interés educativo y social también se ha visto reflejado en el ámbito científico, que ha prestado especial atención durante las últimas décadas a la mejora de la convivencia escolar, como la mejor vía para mejorar la vida social del alumnado (Ortega, 2015).

Los estudios sobre convivencia reconocen el carácter colectivo y dinámico del constructo, que se construye en torno a las interacciones entre alumnado, profesorado y familias. Este entramado de relaciones interpersonales que caracteriza la vida en los centros educativos conforma las creencias, actitudes y valores de sus protagonistas e influye lógicamente en la calidad del proceso de enseñanza y aprendizaje (García-Raga y López-Martín, 2009; Godás, Santos y Lorenzo, 2008; Viguer y Solé, 2011). Entender la convivencia escolar como un constructo colectivo, intergrupal y dinámico supone tener en cuenta los aspectos positivos que conlleva el establecimiento de vínculos interpersonales, pero también prestar atención a las dificultades que surgen en las relaciones, siendo el bullying y los subsecuentes problemas de victimización y agresión entre iguales de los más preocupantes dentro de la escuela (Modecki, Minchin, Harbaugh, Guerra y Runions, 2014).

Ser maltratado, atacado, golpeado o, en definitiva, victimizado por los iguales, ha sido el problema de la convivencia escolar más ampliamente investigado en los últimos años (Baldry y Farrington, 2007; Ttofi y Farrington, 2011). Cuando estas conductas suponen agresiones y situaciones de victimización en las que uno o varios escolares intencional y repetidamente en el tiempo ejercen su poder físico, psicológico o social contra una víctima que difícilmente puede defenderse por sí misma, se entienden como dinámicas de acoso escolar (bullying, en su expresión inglesa) (Olweus, 1999). Pero también preocupan las conductas agresivas y de victimización que pueden vivirse de forma transitoria, que sin basarse en un esquema de dominio y sumisión estable (dinámicas pre-bullying), pueden ser precursoras del bullying, y desde luego, están asociadas a consecuencias psicológicas, sociales y emocionales igualmente dañinas para sus implicados (Crick y Grotpeter, 1995; Monks y Smith, 2010). 
La investigación sobre bullying en las escuelas de todo el mundo indica que un importante número de escolares se implica de prolongada, persistente y cruel en estos problemas. Para muchos de ellos (entre el 20 y el 35\%) el fenómeno bullying puede no durar mucho tiempo, disolverse relativamente pronto, pero entre un 5-8\% mantiene la agresión o la victimización de forma tan prolongada y sus efectos son tan devastadores que podemos hablar de fenómenos de victimización y agresión impune. Al menos un 5\% del alumnado de los cursos centrales de la enseñanza primaria y los primeros de la secundaria obligatoria, se encuentra envueltos en estos problemas con una periodicidad semanal y ese mismo porcentaje de alumnado agrede a otros/as al menos una vez a la semana, si atendemos a las tendencias generales de los estudios con muestras nacionales (García-Fernández, Romera y Ortega, 2016; Ramírez y Justicia, 2006) y europeas (Livingston,Haddon, Görzig y Ólafsson, 2011; Smith, 2016; Watters, 2011).

Efectivamente, el acoso está presente con distintos niveles de gravedad que se relacionan con la evolución concreta de su aparición en un centro o aula. Ello nos hace hablar de grados de gravedad, desde una tipología menos grave, pero ampliamente presente entre los escolares (entre el 20 y 35\%), a otra más grave, muy dañina, menos extendida y que cursa con un gran riesgo de producir serios daños en la personalidad de quien lo sufre y que es indicador de grave perturbación psicológica en la personalidad de quien lo infringe (Juvonen y Graham, 2014; Ortega, 2010).

Los estudios sobre prevalencia en agresión y victimización señalan que la implicación es más alta en los niños que en las niñas, tanto en el caso de la victimización como en el de la agresión (Cerezo, Sánchez, Ruíz, y Arense, 2015; Menesini y Salmivalli, 2017; Romera et al., 2017). En cuanto a la edad, el momento de mayor incidencia de conductas de maltrato entre escolares se sitúa entre los 11 y los 15 años, coincidiendo con el tercer ciclo de la educación primaria y los primeros cursos de la secundaria obligatoria en España (Garaigordobil y Oñederra, 2009; García-Fernández, Romera, y Ortega, 2015; Hymel y Swearer, 2015). En esta línea se ha observado que entre las variables de carácter personal, la autoestima está presente en la mayoría de estudios de acoso escolar en los que se reconoce una diferenciación para los comportamientos de victimización y agresión injustificada (Fanti y Henrich, 2015; Modecki, Barber, y Vernon, 2013; Suresh y Tipandjan, 2012) en el sentido general de una más baja autoestima de los escolares que se sienten victimizados por otros (Garaigordobil, Martínez- 
Valderrey, y Aliri, 2013; Suresh y Tipandjan, 2012). Aunque, en algunas investigaciones esta relación de influencia se ve mediada por el género, siendo la baja autoestima relacionada con la victimización en las chicas y la alta autoestima con los comportamientos de agresión en los chicos (Brito y Oliveira, 2013).

Las investigaciones sobre las características de los implicados en formas graves de bullying (victimización y agresión) han descrito además distintos factores protectores y de riesgo relacionados con el contexto. En el contexto social, la falta de ajuste en la red de iguales ha sido señalada como factor de riesgo en la implicación en los fenómenos de agresión y victimización (Berger y Caravita, 2016; Rigby, 2003). Concretamente se reconoce que quienes desarrollan comportamientos de victimización presentan un mal ajuste social entre los compañeros y compañeras y son señalados como un grupo que tiende al aislamiento (Cerezo, Sánchez-Lacasa, Ruiz-Esteban, y Arense, 2015; Cook, Williams, Guerra, Kimy Sadek, 2010; Holt, Finkelhor, y Kantor, 2007; Estévez, Murgui y Musitu, 2009). Pero también, se ha reconocido que la calidad de las relaciones positivas se relaciona con una reducción de los efectos negativos en la victimización (Woods, Doane y Kalsi, 2009). En el caso de los comportamientos de agresión, las investigaciones han demostrado que sus protagonistas pueden poner en práctica ciertas habilidades sociales que les permiten actuar impunemente y conseguir la aceptación del grupo (Berger y Caravita, 2016; Gini, Pozzoli y Hauser, 2011).

El ajuste a las normas sociales es otro elemento contextual de interés científico para la comprensión de la agresión y la victimización (Del Rey, Casas y Ortega-Ruiz, 2017; OrtegaRuiz, Del Rey y Casas, 2013). Se han identificado dificultades para aceptar las normas sociales en aquellos que desarrollan comportamientos de agresión (Cerezo, 2002). En victimización, se ha definido a estos escolares como sumisos a las normas impuestas (Ortega, 2000), aunque también se les reconocen comportamientos de incumplimiento de las normas (Serrate, 2007). Estudios más recientes han encontrado una disminución del riesgo de ser victimizado en aquellos contextos en los que el profesorado desaprueba las conductas de indisciplina (Saarento, Kärnä, Hodges y Salmivalli, 2013), siendo un respaldo positivo las normas de aula para favorecer la calidad de las relaciones personales (Herrera, Romera, Ortega, y Gómez, 2016). Pero también, Saarento, Boulton y Salmivalli (2015) demostraron cambios en las percepciones de los estudiantes sobre la intimidación que hacían disminuir dichas conductas, mediados por los efectos de programas implantados, con respecto a las actitudes de aprobación o desaprobación de sus profesores respecto de comportamientos de intimidación. Además la per- 
cepción sobre la equidad de las normas se ha relacionado de manera significativa con una mayor participación y rendimiento académico de los estudiantes y con niveles menores de disruptividad, agresión y victimización (Gottfredson, Gottfredson, Payne y Gottfredson, 2005).

La literatura científica sobre victimización y agresión entre iguales, en el marco de estudios sobre bullying, ha documentado la relevancia de factores contextuales claves para la comprensión de la complejidad de estos fenómenos (Casas, Del Rey y Ortega, 2013; Fanti, Demetruiou y Hawa, 2012; García-Fernández, Romera y Ortega, 2015; Hemphill et al., 2012; Hinduja y Patchin, 2013; Preddy y Fite, 2012) tanto como la relevancia de factores personales (Atik y Güneri, 2013; Feslt y Quandt, 2013; García-Fernández et al., 2015).

La prevalencia de los niveles de agresión y victimización entre escolares y las consecuencias negativas asociadas a los mismos, ponen de manifiesto hasta qué punto es importante la erradicación y la prevención de los mismos para mejorar la convivencia y el clima escolar (Ttofi y Farrington, 2011). Por ello, el objetivo del trabajo que aquí se presenta ha sido conocer los factores que estarían explicando la implicación en los fenómenos de victimización y agresión injustificada entre escolares.

\section{Objetivos e hipótesis}

El objetivo de este estudio ha sido conocer los factores protectores y de riesgo, tanto de carácter personal como de tipo contextual, que estarían explicando la implicación en los fenómenos de victimización y agresión injustificada entre escolares. Partimos de la hipótesis de que tanto los factores personales como contextuales influyen en la implicación en victimizacióny agresión, a pesar de que la tendencia de las investigaciones se ha dirigido a prestar una mayor atención a las variables psicológicas de los individuos (García-Fernández et al., 2015).

\section{Método}

\section{Participantes}

Han participado 1278 estudiantes (52.3\% niños y $47.7 \%$ niñas) de los últimos cursos de educación primaria (Grados $5^{\circ}$ y $6^{\circ}$ ) de centros públicos, privados y concertados de las ocho provincias andaluzas. El $49.4 \%$ estaba matriculado en $5^{\circ}$ curso de primaria y el $50.6 \%$ en 
$6^{\circ}$ curso. Las edades de los escolares estaban comprendidas entre los 10 y los 14 años $(M=$ $11.11 ; D T=.75)($ Ver Tabla 1$)$.

Tabla 1. Distribución de la muestra del alumnado por curso

\begin{tabular}{lllll}
\hline & \multicolumn{3}{c}{$5^{\text {o }}$ Primaria } & \multicolumn{2}{c}{$6^{\text {a Primaria }}$} \\
\cline { 2 - 5 } & $\mathrm{N}$ & $\%$ & $\mathrm{~N}$ & $\%$ \\
\cline { 2 - 5 } Chicos & 334 & 26.0 & 338 & 26.4 \\
\hline Chicas & 297 & 23.4 & 309 & 24.2 \\
\hline
\end{tabular}

\section{Instrumentos}

Se ha usado una batería de instrumentos entre los que se encuentra la Escala de Convivencia Escolar (ECE) (Del Rey, Casas y Ortega- Ruiz, 2017), que evalúa diferentes aspectos relativos a la convivencia escolar desde la percepción del alumnado, desde la percepción de seguridad y protección, hasta la forma en que él/ella cree que se cumple la disciplina o la forma en que él/ella misma asume las normas y su satisfacción al respecto de todas estas cuestiones (Del Rey, Casas y Ortega-Ruiz, 2017). Esta escala está compuesta por 50 ítems tipo Likert formulados en positivo, con 5 opciones de respuesta que hacen referencia a la frecuencia de percepción, con valores que van desde $0=$ nunca, hasta $4=$ siempre. Estos ítems se agrupan en ocho dimensiones del constructo convivencia escolar. Para este estudio se analizan las siguientes dimensiones, ateniéndonos a la revisión de la literatura expuesta:

Victimización $(\alpha=.81)$ : percepción del alumnado de estar expuesto a acciones negativas violentas por parte de otro u otros estudiantes. Compuesta de seis ítems. Ej. Algún/a compañero/a me ha golpeado.

Disruptividad $(\alpha=.79)$ : acciones negativas llevadas a cabo por el alumnado y que interrumpen el proceso de enseñanza-aprendizaje. Compuesta por seis ítems. Ej. Hay niños/as que no dejan dar clase.

Red Social de Iguales $(\alpha=.77)$ : fortalezas del microsistema de los iguales que fomentan el desarrollo personal y socio-emocional del alumnado. Se compone de nueve ítems. Ej. Los/as alumnos/as nos llevamos bien.

Agresión $(\alpha=.78)$ : conductas hostiles llevadas a cabo por el alumnado hacia sus compañeros/as. Compuesta por cuatro ítems. Ej. He insultado a algún/a compañero/a. 
Ajuste normativo $(\alpha=.72)$ : adecuación de las conductas y los comportamientos del alumnado a las normas y convenciones del aula y el centro escolar. Lo constituyen cinco ítems. Ej. Cumplo las normas.

Indisciplina $(\alpha=.71)$ : acciones del alumnado contrarias a las normas de convivencia de aula y de centro. La componen cuatro ítems. Ej. Sólo cumplo las normas que me convienen.

Para medir la autoestima se ha utilizado la escala sobre autoestima de Rosenberg (1965), compuesta por diez ítems, unos formulados en negativo y otros en positivo, que describen la confianza frente a la desconfianza, el pesimismo y el fatalismo que los escolares depositan en sí mismos. Las dos dimensiones que valora esta escala se evalúan mediante ítems tipo Likert de 4 opciones de respuesta que indican el grado de acuerdo o desacuerdo con las afirmaciones que se presentan siendo 1 "totalmente de acuerdo" y 4 "totalmente de acuerdo". Estas dimensiones distinguen entre autoestima positiva conformada por seis ítems $(\alpha=$ .716), y otra la autoestima negativa compuesta por cuatro ítems ( $\alpha=.647)$ (GarcíaFernández, Romera y Ortega, 2015).

\section{Procedimiento}

La selección de la muestra se llevó a cabo mediante un procedimiento de muestreo aleatorio estratificado (Contandriopoulos, Champagne, Potvin Denis y Boyle, 1991) bajo los criterios de curso y provincia: dos cursos $\left(5^{\circ}\right.$ y $6^{\circ}$ de Educación Primaria), por 8 provincias (total 16 estratos). Con un error muestral del 3\% y el nivel de confianza del 97\% (Santos et al., 2003).

Se contactó con los centros seleccionados de forma aleatoria mediante un documento formal donde se indicaba la naturaleza, la finalidad y los objetivos de la investigación. Una vez que el centro aceptó la participación y las familias fueron informadas, los investigadores formados para ello, se trasladaron a los centros educativos con el fin de administrar los cuestionarios teniendo en cuenta la disponibilidad horaria y el número de aulas. Se enfatizó en la naturaleza voluntaria y anónima del mismo. Los profesores responsables del aula se ausentaron durante su administración que se hizo de manera colectiva y en una única sesión de 50 minutos. 


\section{Análisis de datos}

Se realizaron análisis descriptivos de tendencia central y de dispersión (Buendía, Colás y Hernández, 1998) y la regresión lineal múltiple (Aldrich y Nelson, 1984) para establecer las variables predictoras de la victimización y agresión injustificada.

Para el estudio descriptivo se utilizaron análisis univariados: tales como la prueba $\mathrm{T}$ de Student y las correlaciones de Spearman. Para el estudio de las relaciones de influencia de cada una de las variables estudiadas sobre los perfiles de victimización y agresión se realizaron los análisis de regresión logística lineal mediante el método de pasos sucesivos (Aldrich y Nelson, 1984), en los que se consideraron los niveles de significación de $p<.05$ y $p<.01$.

Para registrar y codificar los datos se utilizó el programa estadístico SPSS en su versión 20.0 .

\section{Resultados}

Los análisis descriptivos indicaron medias más elevadas en victimización $(M=1.075$; $D T=.937)$ que en agresión $(M=.891 ; D T=.833)$.Se observaron diferencias estadísticamente significativas en función de la variable género en la implicación en victimización y agresión. En concreto, los resultados de la prueba $\mathrm{T}$ para la igualdad de medias mostraron tanto en victimización [t (3.276)=.295; $p=.001 ; d=.847]$ como en agresión $[\mathrm{t}(8.994)=28.876 ; p=$ $.000 ; d=.7555]$ que los chicos presentaron medias más elevadas que las chicas (ver Tabla 2 ).

Tabla 2. Estadísticos descriptivos de victimización y agresión según el género

\begin{tabular}{llllll}
\hline Estadísticos de grupo & \multicolumn{5}{l}{} \\
\hline \multirow{2}{*}{ Victimización } & Género & $\mathrm{N}$ & $\mathrm{M}$ & D.T. & E.T \\
\cline { 2 - 6 } & Hombre & 581 & .998 & .849 & .035 \\
\multirow{2}{*}{ Agresión } & Mujer & 534 & .832 & .842 & .036 \\
& Hombre & 605 & .979 & .836 & .034 \\
& Mujer & 561 & .585 & .654 & .027 \\
\hline
\end{tabular}


En relación a la edad, medida por el curso académico ( $5^{\circ} \mathrm{Grado}, 10$ y 11 años; $6^{\circ} \mathrm{Gra}-$ do, 11 y 12 años; también se encontraron diferencias estadísticamente significativas en la conducta de agresión. Los análisis descriptivos de comparaciones de medias mediante la prueba $\mathrm{T}$ mostraron que en el fenómeno de agresión $[\mathrm{t}(-.925,1169)=5.583 ; p=.004 ; d=$ .780], existen medias más altas en $6^{\circ}$ que en el $5^{\circ}$ curso de primaria (ver Tabla 3 ). No se observaron diferencias estadísticamente significativas para la victimización.

Tabla 3. Estadísticos descriptivos de victimización y agresión según el curso

\begin{tabular}{llllll}
\hline Estadísticos de grupo & \multicolumn{6}{l}{} \\
\hline & Curso & $\mathrm{N}$ & $\mathrm{M}$ & D.T. & E.T \\
\hline Victimización & $5^{\mathrm{o}}$ & 551 & .901 & .831 & .035 \\
& $6^{\mathrm{o}}$ & 573 & .944 & .874 & .036 \\
\multirow{2}{*}{ Agresión } & $5^{\circ}$ & 578 & .724 & .737 & .030 \\
& $6^{\mathrm{o}}$ & 599 & .856 & .817 & .033 \\
\hline
\end{tabular}

Las correlaciones de Pearson señalaron diferencias estadísticamente significativas en todas las variables estudiadas, tanto en victimización como en agresión. El ajuste social entre iguales, el ajuste a las normas sociales y la autoestima positiva mostró una correlación relación inversa, lo que indica que a medida que aumenta la implicación en victimización y agresión disminuyen estas variables (ver Tabla 4). En cuanto a las variables indisciplina, disruptividad y autoestima negativa mostró una correlación positiva o directa, lo que implica que a medida que aumenta la victimización y la agresión también lo hacen estas variables.

Tabla 4. Correlaciones de Pearson para Victimización y Agresión Injustificada

\begin{tabular}{llllll}
\hline & $\begin{array}{l}\text { Ajuste } \\
\text { social con } \\
\text { los iguales }\end{array}$ & $\begin{array}{l}\text { Ajuste a las } \\
\text { normas so- } \\
\text { ciales }\end{array}$ & $\begin{array}{l}\text { Indisciplina y } \\
\text { disruptividad }\end{array}$ & Autoestima + & Autoestima - \\
\hline Victimización & $-.496 *$ & $-.254^{*}$ & $.270^{*}$ & $-.199 *$ & $.357^{*}$ \\
Agresión & $-.339 *$ & $-.544^{*}$ & $.568^{*}$ & $-.139 *$ & $.167^{*}$ \\
\hline
\end{tabular}

*Correlaciones mayores a 0.4 . 
Los resultados de los análisis de regresión lineal fueron estadísticamente significativos para la victimización $(F=95.789 ; g l=4)$. El valor de $\mathrm{R}^{2}$ fue de .326 . El ajuste social está negativamente relacionado con la victimización, por el contrario, las variables de ajuste a las normas, la indisciplina y disruptividad y la autoestima negativa mostraron una relación directa con la victimización (ver Tabla 5).

Tabla 51. Modelo de regresión lineal para la victimización II

\begin{tabular}{llll}
\hline \multirow{2}{*}{ Modelo } & & \multicolumn{2}{l}{ Coeficientes no estandarizados } \\
\hline \multirow{2}{*}{1} & & $\beta_{\mathrm{p}}$ & Error típ. \\
\cline { 3 - 4 } & (Constante) & 1.276 & .221 \\
& $\mathrm{X}_{1}$ Ajuste Social & $-.487\left(\beta_{1}\right)$ & .035 \\
$\mathrm{X}_{2}$ Ajuste a las normas socia- & $.131\left(\beta_{2}\right)$ & .055 \\
les & & .044 \\
$\mathrm{X}_{3}$ Indisciplina y disruptivi- \\
dad
\end{tabular}

Para la agresión el modelo de regresión lineal estimó una relación estadísticamente significativa $(F=144.692 ; g l=4)$ que se señala una relación negativa con el ajuste social y el ajuste a las normas, lo que indica que a medida que aumentan estas variables, disminuye la agresión. Por el contrario, la indisciplina y disruptividad indicaron una relación positiva, es decir que a medida que aumenta la disruptividad y la disciplina aumenta también la agresividad injustificada (ver Tabla 6). El valor de $\mathrm{R}^{2}$ fue de .416 lo que indica que aproximadamente la mitad de la variabilidad de la agresividad es explicada por las variables.

Tabla 62. Modelo regresión lineal para la agresividad injustificada

\begin{tabular}{|c|c|c|c|}
\hline \multicolumn{2}{|c|}{ Modelo } & \multicolumn{2}{|c|}{ Coeficientes no estandarizados } \\
\hline & & $\beta_{\mathrm{p}}$ & Error típ. \\
\hline \multirow[t]{4}{*}{1} & (Constante) & 1.962 & .182 \\
\hline & $\mathrm{X}_{1}$ Ajuste Social & $-.176\left(\beta_{1}\right)$ & .029 \\
\hline & $\begin{array}{l}\mathrm{X}_{2} \text { Ajuste a las normas socia- } \\
\text { les }\end{array}$ & $-.274\left(\beta_{2}\right)$ & .047 \\
\hline & $\begin{array}{l}X_{3} \text { Indisciplina y disruptivi- } \\
\text { dad }\end{array}$ & $.445\left(\beta_{3}\right)$ & .038 \\
\hline
\end{tabular}




\section{Discusión y Conclusiones}

La finalidad de esta investigación fue examinar los factores protectores y de riesgo que estarían explicando los fenómenos de victimización y agresión injustificada y pertinaz entre iguales en escolares de primaria. Desde un punto de vista dinámico de los comportamientos de violencia interpersonal y muy particularmente la agresión injustificada y pertinaz que provoca victimización, se pretende conocer la existencia de relaciones explicativas comunes, en función de ciertas variables personales (de género, edad así como de autoestima positiva o negativa) y de las características contextuales o aspectos concretos que afectan al contexto social inmediato en el cual tienen lugar las relaciones interpersonales de los iguales. Partimos de la hipótesis de que tanto los factores personales como contextuales influyen en la implicación en la percepción de victimización y la agresión, a pesar de que como se ha señalado la tendencia de las investigaciones se ha dirigido a prestar una mayor atención a las variables psicológicas de los individuos (García-Fernández et al., 2015).

Las investigaciones sobre el tipo de factores que influyen en los comportamientos característicos del bullying han mostrado resultados que identifican tanto factores contextuales como de tipo personal (Atik y Güneri, 2013; Casas, Del Rey y Ortega, 2013). Los resultados del presente trabajo señalan que además de a los factores personales de los escolares implicados, se ha de prestar una particular atención a los factores contextuales para comprender por qué los niños y niñas se implican, o se perciben implicados, en estas formas graves de bullying. Ello es coherente con ciertos estudios internacionales (Demetruiou y Hawa, 2012; Hinduja y Patchin, 2013; Preddy y Fite, 2012) así como con nuestros propios estudios previos (García-Fernández et al., 2015). Quizás debemos entender que el agravamiento de ambos comportamientos (agresión injustificada y victimización) que son las dos conductas que definen el bullying, tiene lugar no solo por ciertas características de los protagonistas, sino también como expresión de elementos contextuales que son importantes, como la indisciplina, el desajuste normativo o el pertinaz desajuste de la propia red de iguales (ajuste social). . Estos elementos resultaN importantes como factores del contexto social inmediato, de la convivencia escolar y están al alcance de los docentes y los responsables de las escuelas. Hay que tenerlos en cuenta si se desea prevenir no solo la aparición, sino también el agravamiento de las conductas que constituyen el fenómeno bullying. 
Los resultados sobre los factores estudiados permiten identificar cómo influyen en los perfiles de victimización y agresión injustificada. Respecto del género, se han observado diferencias que identifican a los chicos como el grupo más implicado, hecho que coincide con la mayoría de las investigaciones de este campo (Ortega, 2010; Cerezo et al., 2015; Menessini y Salmivalli, 2017; Romera, et al., 2017). No obstante, los estudios que inciden en dichas diferencias hacen referencia igualmente al efecto que pueden tener otras variables como la autoestima (Brito y Oliveira, 2013) o el estatus sociométrico y la popularidad (Sentse, Kretschmer y Salmivalli, 2015). En lo que a la edad se refiere, no se han encontrado diferencias aunque se constata un mayor nivel de casos conforme el alumnado asciende en los cursos que, de acuerdo con investigaciones previas, alcanzaría su culmen en los primeros cursos de la Educación Secundaria Obligatoria y, a partir de ahí, irían disminuyendo progresivamente (Garaigordobil y Oñederra, 2009), quizás debido al corto rango de edad del estudio.

Los comportamientos de victimización, como era esperado, son asociados con una baja autoestima, en consonancia con los estudios en los que han sido caracterizadas con un menor nivel de valoración personal en las víctimas (Garaigordobil, Martínez-Valderrey y Aliri, 2013; Suresh y Tipandjan, 2012). Respecto del ajuste social entre iguales, en el presente estudio la victimización queda explicada por el bajo nivel de este factor, en coherencia con los estudios previos que reconocen en las víctimas un mal ajuste social (Cerezo, Sánchez- Lacasa, Ruiz-Esteban y Arense, 2015), quizás debido a dificultades en asertividad de ellas y, por supuesto, al déficit de empatía que suele caracterizar al agresor. Por último, se señala una relación entre victimización y ajuste normativo siendo que las víctimas mostrarían conductas más ajustadas a las normas, como se ha puesto de manifiesto por Díaz-Aguado y Martínez-Arias (2013). Estos hallazgos indican que el contexto social próximo de los escolares, su baja autoestima pueden suponer elementos de riesgo para el desarrollo de la victimización, tal y como hemos puesto de manifiesto en estudios previos (García- Fernández et al., 2015).

En el caso del fenómeno de la agresividad injustificada, se ha señalado también como factor de riesgo el bajo ajuste social, relación que puede ser explicada atendiendo a las emociones negativas que despiertan estas conductas entre los iguales, siguiendo la línea de estudios previos que reconocen a los agresores con escasas habilidades, pero con una cierta habilidad social que les permite conseguir la aceptación del grupo (Berger y Caravita, 2016; Gini, Pozzoli y Hauser, 2011). Respecto al ajuste normativo, en este estudio se reconoce que los 
escolares que desarrollan comportamientos de agresión injustificada presentan también comportamientos disruptivos y ajuste a las normas, lo que indica la importancia de la regulación de las relaciones interpersonales (Herrera, Romera, Ortega y Gómez-Ortiz, 2016). Esta relación puede ser explicada en base al ajuste social, ya que esa escasa habilidad para gestionar sus relaciones sociales con los iguales puede ser un factor que explique los comportamientos desarrollados en otros contextos en los que también deben desarrollar habilidades sociales. Estos resultados vuelven a reforzar la idea del que las dinámicas de agresión y victimización cobran especial importante enmarcadas en un contexto social.

De la aportación de los hallazgos del presente estudio a este ámbito de conocimiento podemos extraer una serie de implicaciones prácticas relacionadas con la prevención pero sobre todo de la intervención educativa en fenómenos muy severos de bullying. Los datos sugieren la necesidad de prestar una mayor atención a los factores de carácter contextual en la configuración de ambas procesos, la agresión injustifica y la victimización Los factores de ajuste social entre iguales y ajuste normativo pueden ser claves para explicar las situaciones de dominio-sumisión (Ortega, 2000 y 2010) que se generan en el seno de la convivencia escolar y que son tipos graves de bullying. Que el docente sepa afrontar situaciones de acoso y cuente con recursos para prevenirlas se convierte en una prioridad, no solo para garantizar un clima social positivo que facilita el proceso de enseñanza-aprendizaje, sino porque es evidente el efecto positivo de las relaciones interpersonales en el desarrollo psicológico y social, lo cual ayudará a formar a ciudadanos competentes para vivir y gestionar su vida en sociedad. Abordar estos problemas requiere tener en cuenta planteamientos preventivos enfocados sobre todo al desarrollo y puesta en práctica de habilidades sociales y emocionales que ayuden alos escolares a integrarse en su entorno social, estimulando actitudes, valores y comportamientos para prevenir los problemas de violencia en las diferentes etapas educativas (GarcíaFernández, Gómez-Ortiz y Romera, 2016).

Es necesario igualmente finalizar haciendo referencia a las limitaciones que pueda presentar la investigación planteada. La medición de las variables a través de autoinforme supone que los resultados puedan contemplar cierta deseabilidad social expresada por los sujetos. Sería necesario incluir la percepción de otros grupos para evaluar otras variables contextuales como la aceptación social, la popularidad o las metas sociales, así como datos cualitativos que permitan contemplar la perspectiva de los espectadores. Como futura línea de in- 
vestigación se plantea el estudio longitudinales y de modelos explicativos para analizar la estabilidad de estos hallazgos.

\section{Agradecimientos}

Este trabajo ha sido finaciado por el Ministerio de Economía, Industria y Competitividad (número PSI2016-74871-R).

\section{Referencias}

Aldrich, J. y Nelson, F. (1984). Linear probability, logit and probit models. Beverly Hills, CA: Sage.

Atik, G., y Güneri, O. Y. (2013). Bullying and victimization: Predictive role of individual, parental, and academic factors. School Psychology International, 34(6), 658-673. doi: $10.1177 / 0143034313479699$.

Baldry, A. C. y Farrington, D. P. (2007).Effectiveness of programs to prevent school bullying. Victims and Offenders, 2(2), 183-204. doi: 10.1080/15564880701263155.

Berger, C. y Caravita, C.S. (2016). Why do early adolescents bully? Exploring the Influence of Prestige Norms on Social and Psychological Motives to Bully. Journal of Adolescence, 46, 45-56. doi:10.1016/201510020

Brito, C. C. y Oliveira, M. T. (2013).Bullying and self-esteem in adolescents from public schools. Jornal de Pediatria, 89(6), 601-607. doi:10.1016/j.jped.2013.04.001

Buendía, L., Colás, P., y Hernández, F. (1998). Métodos de investigación en psicopedagogía. Madrid: McGraw Hill.

Burton, K. A., Florell, D., y Wygant, D. B. (2013). The role of peer attachment and normative beliefs about aggression on traditional bullying and cyberbullying. Psychology in the Schools, 50(2), 103-115. doi:10.1002/pits.21663

Casas, J. A., Del Rey, R., y Ortega-Ruiz, R. (2013). Bullying and cyberbullying: Convergent and divergent predictor variables. Computers in Human Behavior, 29(3), 580-587. doi:10.1016/j.chb.2012.11.015

Cerezo, F. (2002). El bullying y su relación con las actitudes de socialización en una muestra de adolescentes. Revista Electrónica Interuniversitaria de Formación del Profesorado, 5 (1), 1-8. Disponible en: https://dialnet.unirioja.es/ejemplar/100558

Cerezo, F., Sánchez-Lacasa, C., Ruiz-Esteban, C., y Arense, J. J. (2015). Roles en bullying de adolescentes y preadolescentes, y su relación con el clima social y los estilos educati- 
vos parentales. Revista de Psicodidáctica, 20(1), 139-155. doi: 10.1387/RevPsicodidact.11097

Contandriopoulos, A. P., Champagne, F., Potvin, L., Denis, J. L., y Boyle, P. (1991). Preparar un proyecto de investigación. Barcelona: SG Editores.

Cook, C. R., Williams, K. R., Guerra, N. G., Kim, T. E., y Sadek, S. (2010). Predictors of bullying and victimization in childhood and adolescence: A meta-analytic investigation. School Psychology Quarterly, 25(2), 65-83. doi: 10.1037/a0020149

Crick, N. y Grotpeter, J. (1995). Relational Aggression, gender, and social psychological adjustment. Child Development, 66 (3), 710-722. doi:10.2307/1131945

Del Rey, R., Casas, J. A., y Ruiz, R. O. (2017). Desarrollo y validación de la Escala de Convivencia Escolar. Universitas Psychologica, 16(1), 1-12. doi: 10.11144/Javeriana.upsy16-1.dvec

Delgado-Gallego, I., Oliva, A., y Sánchez Queija, I. (2011). Apego a los iguales durante la adolescencia y la adultez emergente. Anales de Psicología, 27(1), 155-163. Disponible en: http://www.redalyc.org/articulo.oa? $\mathrm{Id}=16717018018$

Díaz-Aguado, M. J. y Martínez Arias, R. (2013). Peer bullying and disruption-coercion escalations in student-teacher relationship. Psicothema, 25(2), 206-213. doi: 10.7334/psicothema2012.312

Estévez, E., Murgui, S., y Musitu, G. (2009).Psychological adjustment in bullies and victims of school violence.European Journal of Psychology of Education, 24(4), 473-483. doi:10.1007/BF0317876

Fanti, K. A. y Henrich, C. C. (2015). Effects of Self-Esteem and Narcissism on Bullying and Victimization During Early Adolescence. The Journal of Early Adolescence, 35 (1), 529. doi: $10.1177 / 0272431613519498$

Fanti, K. A., Demetriou, A. G., y Hawa, V. V. (2012). A longitudinal study of cyberbullying: Examining risk and protective factors. European Journal of Developmental Psychology, 9(2), 168-181. doi:10.1080/17405629.2011.643169

Festl, R. y Quandt, T. (2013). Social relations and cyberbullying: The influence of individual and structural attributes on victimization and perpetration via the Internet. Human Communication Research, 39(1), 101-126. doi: 10.1111/j.1468-2958.2012.01442.x

Garaigordobil, M. y Oñederra, J. A. (2009). Un análisis del acoso escolar desde una perspectiva de género y grupo. Ansiedad y Estrés, 15(2-3), 193-205. Disponible en: http://www.sc.ehu.es/ptwgalam/art_completo/2009/A\&E\%20acoso\%202009.pdf 
Garaigordobil, M., Martínez-Valderrey, V., y Aliri, J. (2013).Autoestima, empatía y conducta agresiva en adolescentes víctimas de bullying presencial. European Journal of Investigation in Health, Psychology and Education, 3(1), 29-40. doi: 10.1989/ejihpe.v3i1.21

García-Fernández , C. M. , Romera, E. M. R., y Ortega, R. (2015). Explicative factors of faceto-face harassment and cyberbullying in a sample of primary students. Psicothema, 27(4), 347-353. doi: 10.7334/psicothema2015.35

García-Fernández, C. M., Romera-Félix, E. M., y Ortega-Ruiz, R. (2016). Relations between Bullying and Cyberbullying: Prevalence and Co-ocurrence. Pensamiento Psicológico, 14(1), 49-61. doi:10.11144/Javerianacali.PPSI14-1.rbcp

García-Fernández, C., Gómez-Ortiz, O., y Romera, E. (2016). La dinámica del bullying: Afrontar y prevenir el problema. En Córdoba- Alcaide, F., Ortega- Ruíz, R., y NeilKroyer, O. (2016). Gestión de la convivencia y afrontamiento de la conflictividad escolar y el bullying. Chile: Ril editores.

García-Raga, L. y López-Martín, R. (2009). Convivir en la escuela. Una propuesta para su aprendizaje por competencias. Revista de Educación, 356, 531-555. doi:104438/1988592X-RE-2011-356-050

Gini, G., Pozzoli, T., y Hauser, M. (2011). Bullies have Enhanced Moral Competence to Judge relative to Victims, but Lack Moral Compassion. Personality and Individual Differences, 50, 603-608. doi:10.1016/j.paid.2010.12.002

Godás, A., Santos, M. A., y Lorenzo, M. (2008). Convivir en los centros educativos. ¿Cómo lo ven los alumnos, padres y profesores?. Bordón, 60 (1), 41-58. Disponible en: <https://recyt.fecyt.es/index.php/BORDON/article/view/28835/15380>

Gottfredson, G. D., Gottfredson, D. C., Payne, A. A., y Gottfredson, N. C. (2005). School climate predictors of school disorder: Results from a national study of delinquency prevention in schools. Journal of Research in Crime and Delinquency, 42, 412-444.doi: 10.1177/0022427804271931

Hemphill, S. A., Kotevski, A., Tollit, M., Smith, R., Herrenkohl, T. I., Toumbourou, J. W., y Catalano, R. F. (2012).Longitudinal predictors of cyber and traditional bullying perpetration in Australian secondary school students. Journal of Adolescent Health, 51(1), 59-65. doi: 10.1016/j.jadohealth.2011.11.019

Herrera, M., Romera, E. M., Ortega, R., y Gómez-Ortiz, O. (2016).Influence of social motivation, self-perception of social efficacy and normative adjustment in the peer setting. Psicothema, 28(1), 32-39. doi: 10.7334/psicothema2015.135 
Hinduja, S. y Patchin, J. W. (2013).Social influences on cyberbullying behaviors among middle and high school students. Journal of Youth and Adolescence, 42(5), 711-722. doi: $10.1007 / \mathrm{s} 10964-012-9902-4$

Holt, M. K., Finkelhor, D., y Kantor, G. K. (2007). Multiple victimization experiences of urban elementary school students: Associations with psychosocial functioning and academic performance. Child Abuse \& Neglect, 31(5), 503-515. doi: 10.1016/j.chiabu.2006.12.006

Hymel, S. y Swearer, S. M. (2015). Four decades of research on school bullying: An introduction. American Psychologist, 70, 293-299. doi: 10.1037/a0038928

Juvonen, J., y Graham, S. (2014). Bullying in schools: The power of bullies and the plight of victims. Annual Review of Psychology, 65, 159-185. doi:10.1146/annurev-psych010213-115030

Liu, J., Bullock, A., y Coplan, R. J. (2014).Predictive relations between peer victimization and academic achievement in Chinese children. School Psychology Quarterly, 29 (1), 89. doi: $10.1037 / \mathrm{spq} 0000044$.

Livingstone, S., Haddon, L., Görzig, A., y Ólafsson, K. (2011).Risks and safety on the internet: The perspective of European children. Full Findings. LSE, London: EU Kids.

Menesini, E. y Salmivalli, C. (2017). Bullying in schools: the state of knowledge and effective interventions. Psychology, Health \& Medicine, 22(1), 240-253. doi: $10.1080 / 13548506.2017 .1279740$

Modecki, K. L., Barber, B. L., y Vernon, L. (2013). Mapping developmental precursors of cyber-aggression: Trajectories of risk predict perpetration and victimization. Journal of Youth and Adolescence, 42(5), 651-661. doi:10.1007/s10964-013-9938-0

Modecki, K. L., Minchin, J., Harbaugh, A. G., Guerra, N. G., y Runions, K. C. (2014). Bullying prevalence across contexts: a meta-analysis measuringcyber and traditional bullying. Journal of Adolescent Health, 55(5), 602-611. doi: 10.1016/j.jadohealth.2014.06.007

Monks, C. y Smith, P. (2010). Peer, self and teacher nominations of participant roles taken in victimization by 5- and 8-year-olds. Journal of Aggression, Conflict and Peace Research, 2, 4-14. doi: 10.5042/jacpr.2010.0532

Olweus, D. (1999). Norway. En P. K. Smith, Y. Morita, J. Junger-Tas, D.Olweus, R. Catalano, y P. Slee (Eds.), The Nature of School Bullying: A cross-national Perspective (pp. 28-48). London: Routledge. 
Ortega, R. (2000). Educar la convivencia para prevenir la violencia. Madrid: Aprendizaje.

Ortega, R. (2010). Agresividad Injustificada, Bullying y Violencia Escolar (pp. 33-54). Madrid: Alianza Editorial.

Ortega, R. (2015). Convivencia y Ciberconvivencia. Un Modelo Educativo para la Prevención del Acoso y el Ciberacoso Escolar. Madrid: Machado Libros.

Ortega, R., Del Rey, R., y Sánchez, V. (2012). Nuevas dimensiones de la convivencia escolar y juvenil. Ciberconducta y relaciones en la Red: Ciberconvivencia. Madrid: Observatorio Estatal de la Convivencia Escolar.

Preddy, T. M. y Fite, P. J. (2012).Differential associations between relational and overt aggression and children's psychosocial adjustment. Journal of Psychopathology and Behavioral Assessment, 34(2), 182-190. doi: 10.1007/s10862-011-9274-1

Ramírez, S. y Justicia, F. J. (2006). El maltrato entre escolares y otras conductas-problema para la convivencia. Electronic Journal of Research in Educational Psychology, 4(9), 265-290. Disponible en: http://www.redalyc.org/articulo.oa?id=293122821007

Romera, E., Ortega, R. Del Rey, R., Casas, J. A., Viejo, C., Gómez-Ortiz, O., Córdoba, F., Zych, I., García-Fernández, C., y Luque, R. (2017). Bullying, cyberbullying y dating violence. Estudio de la gestión de la vida social en estudiantes de Primaria y Secundaria en Andalucía'. Actualidad 75, 1-32. Disponible en: https://www.centrodeestudiosandaluces.es/datos/publicaciones/Actualidad75.pdf

Rigby, K. (2003). Consequences of Bullying in Schools.Canadian Journal of Psychiatry, 48(9), 583-590. doi: 10.1177/070674370304800904

Saarento, S., Boulton, A., y Salmivalli, C. (2015). Reducing bullying and victimization: Student- and classroom-level mechanisms of change. Journal of Abnormal Child Psychology, 43, 61-76. doi: 10.1007/s10802-013-9841-x

Saarento, S., Kärnä, A., Hodges, E. V., y Salmivalli, C. (2013).Student-, classroom-, and school-level risk factors for victimization. Journal of School Psychology, 51(3), 421434. doi: 10.1016/j.jsp.2013.02.002

Serrate, R. (2007). Bullying acoso escolar. Guía para entender y prevenir el fenómeno de la violencia en las aulas. Barcelona: Laberinto.

Smith, P. K. (2016). Research on bullying in schools in European countries. En Smith P. K.; Kwak, K., y Toda, Y. (2016).Bullying in Different Cultures: Eastern and Western Perspectives. Cambridge University press, United Kingdom. 
Suresh, S. y Tipandjan, A. (2012).School bullying victimization and college adjustment. Journal of the Indian Academy of Applied Psychology, 38 (1), 68-73. doi: $10.4135 / 36.753117$

Ttofi, M. M. y Farrington, D. P. (2011). Effectiveness of school-based programs to reduce bullying: A systematic and meta-analytic review. Journal of Experimental Criminology, 7(1), 27-56. Disponible en: https://link.springer.com/article/10.1007\%2Fs11292010-9109-1

Viguer, P. y Solé, N. (2011). Debate familiar sobre valores y convivencia: una investigación participativa para implicar a las familias en el análisis y la transformación de su realidad. Cultura y Educación, 23(1), 105-118. doi: 10.1174/113564011794728542

Watters, R. M. (2011).The nature and extent of pupil bullying in schools in the North of Ireland. Bangor, UK: Department of Education for Northern Ireland.

Woods, S., Done, J., y Kalsi, H. (2009). Peer internalizing and externalizing difficulties: The moderating role of friendship quality. Journal of Adolescence, 32, 293-308. doi: 10.1016/j.adolescence.2008.03.005

Gadner, H. (1993). Multiple intelligences. The theory in practice. New York: Basic Books.

González, M.C. (1997). La motivación académica. Sus determinantes y pautas de intervención. Pamplona: Eunsa.

Hernández, P. y García, L.M. (1997). Enseñar a pensar: un reto para los profesores. La Laguna: Tafor.

Justicia, F. (1996). Metacognición y currículum. En J. Beltrán y C. Genovard (eds.), Psicología de la instrucción I. Variables y procesos básicos (pp. 359-381). Madrid: Síntesis.

Nickerson, R. (1999). Enhancing creativity. En R. Sternberg (Ed.). Handbook of creativity (pp.134-1256). New York: Cambridge University Press. 